oe gouter Acta Univ. Sapientiae, Informatica, 6, 2 (2014) 230-251

DOI: 10.1515/ausi-2015-0006

\title{
Statistical complexity and generalized number system
}

\author{
Ágnes FÜLÖP \\ Loránd Eötvös University, Budapest \\ Faculty of Informatics \\ email: fulop@caesar.elte.hu
}

\begin{abstract}
We apply the concept of statistical complexity to understand the dynamical behaviour of the time series by the probability distribution. This quantity allows to distinguish between the random, regular motion and the structural complexity in finite systems. We determined the numerical approximation of the statistical complexity of the Lozi attractor and the generalized number system.
\end{abstract}

\section{Introduction}

In this article we discuss the statistical complexity [25], which provides a description of a finite measured sequence to specify more complicated dynamical structures. It was extended on wide range of sciences [23, 1, 15].

The idea of complexity was introduced in different forms. We mention some of them: algorithmic complexity (Kolmogorov) [22], amount of information about the past required to predict the future (Crutchfield, Young) [7], complexity of finite sequence (Lempel, Ziv) [24].

There are more questions in the real word, where the statistical complexity is applied. We referred some example as more realistic gas of particles [4, 5], the effective method in the hydrological systems [12], the statistical features

Computing Classification System 1998: J.2

Mathematics Subject Classification 2010: 68U20

Key words and phrases: statistical complexity, Shannon entropy, number theory, Lozi map, chaos, strange attractor 
of the behaviour for DNA [36], the earthquake magnitude time series [26], chaotic motion in Logistic map [13], biological application [32].

The notion of statistical complexity is defined by the concept of the information theory i.e. the entropy and the disequilibrium. The Shannon entropy specifies the gain of the information storage in the disordered system and the disequilibrium characterizes the amount of distance from the equiprobability distribution.

While the entropy allows to describe the direction of flow and the Lyapunov exponent characterizes the chaotic orbits, we can not specify the whole strange attractor in the finite dimensional space. The statistical complexity enables to determine the inner structure of the dynamical system and the location of the strange attractor is shown in the parameter space. It is compared with the complexity of generalized number system, which contains periodic paths.

The numerical simulation plays important role in the chaotic motion, because there are numerous problems, which can not be solved analytically.

We calculated the spectrum of statistical complexity of the two dimensional piecewise Lozi map, which is not differentiable and contains chaotic region. It is compared with the statistical complexity of the finite approximation of the set $\mathrm{B}_{\gamma}$ on the lattice.

The structure of the article is the following:

Section 2 contains the introduction of the statistical complexity to consider different measurement. The chaotic motion is described in Section 3. The definition of the generalized number system and the fundamental set are investigated in Section 4. The numerical results are displayed in Section 5.

\section{Complexity}

In this section we introduce the statistical complexity following the effective entropy by P. Grassberger [16] and the main concept by R. López-Ruiz, H. L. Manchini, X. Calbet (1995) [25, 28, 2]. This definition was extended to the generalized statistical complexity measures by M. T. Martin et al. (2006) [29] for different types of entropy and disequilibrium.

We investigate the notation of a measured sequence [14]. Let us denote $y_{1}, \ldots, y_{n}$ the time series, where $y_{i}$ means the measurement of the quantity $y$ at time $t_{i}=t_{0}+i \Delta t$, and the time interval $\Delta t>0 \in \mathbb{R}$.

The $\underline{x}^{(n)}$ denotes the trajectory of length $n$ in $\mathbb{R}^{d}$, which is a time sequent of the measurement. The kth point of the path of length $n$ is denoted by $x_{k}^{(n)}(k=1, \ldots, n)$. We will study the series of $x_{1}^{(n)}, x_{2}^{(n)}, \ldots, x_{n}^{(n)}$ as a time 
sequent. The set $K$ contains the points of some orbits $x_{k}^{(n)}(k=1, \ldots, n)$.

We will apply the notation of symbolic dynamics, because the idea of complexity is more general concept than this application.

There are $M$ different values of measurements. Each path $\underline{x}^{(\mathfrak{n})}$ for a finite $n$ corresponds to symbolic sequence $\mathrm{O}^{(\mathrm{n})}=\left(\mathrm{o}_{1}, \mathrm{o}_{2}, \ldots, \mathrm{o}_{\mathrm{n}}\right)$, where the symbol $o_{k}(k=1, \ldots, n)$ is chosen from the set $\{1, \ldots, M\}$. Let us consider a time series of length $N^{\prime}$, where $N^{\prime}>>n$. Then a given sequent $\mathrm{O}^{(\mathrm{n})}$ appears with probability $\mathrm{P}\left(\mathrm{O}^{(\mathrm{n})}\right)$ along this long series of length $\mathrm{N}^{\prime}$. The unit of the time interval $\Delta t$ equals to a constant in this description.

\subsection{Statistical complexity}

The statistical complexity is based on the probabilistic description of a finite time series, which provides a statistical approximation of the time sequent. We introduce a measure of statistical complexity, which depends on the finite discrete probability distribution.

We define $\mathrm{N}$-system. Let us assume that there are $\mathrm{N}$ different symbol sequences of length $\mathrm{n}\left\{\mathrm{O}_{1}^{(\mathrm{n})}, \ldots \mathrm{O}_{\mathrm{N}}^{(\mathrm{n})}\right\}$, which correspond to the set of discrete probability distribution $\mathrm{P} \equiv\left\{\mathrm{p}_{1}, \ldots \mathrm{p}_{\mathrm{N}}\right\}$, where $\mathrm{p}_{\mathrm{i}}:=\mathrm{P}\left(\mathrm{O}_{i}^{(\mathrm{n})}\right)\left(\sum_{i=1}^{N} p_{i}=1\right)$ and $p_{i}>0$ for all $i$.

The first we have to consider the entropy i.e. some measure of the amount of information stored $\mathcal{H}$ and the disequilibrium $\mathrm{D}$, which corresponds to the distance from the appropriate probability distribution to the equilibrium.

\subsubsection{Measure of entropy and disequilibrium}

The information measure was introduced as a quantity, which depends on a probability distribution $P=\left\{p_{j}, j=1, \ldots, N\right\}$. In the information theory the entropy was investigated as a unique function, which corresponds to the measure of the uncertainty. The statistical complexity is defined by the Shannon entropy [33], therefore we will investigate this form in $\mathrm{N}$-system:

$$
\mathcal{H}=-\sum_{i=1}^{N} p_{i} \log p_{i} .
$$

This quantity $\mathcal{H} \sim 0$, if the symbol sequence $\mathrm{O}_{\mathrm{c}}^{(\mathrm{n})}$ would be almost probable $\left(p_{c} \sim 1\right)$ and other $\mathrm{O}_{i}^{(\mathfrak{n})}$ would be very improbable $\left(\mathrm{p}_{\mathrm{c}} \sim 0\right)$. $\mathcal{H}_{\max }$ notes the maximal value of $\mathcal{H}$, which reaches the uniform probability distribution $p_{e}=\{1 / N, 1 / N, \ldots, 1 / N\}$ i.e. the equiprobability symbol sequence 
$\mathrm{O}_{e}^{(\mathfrak{n})}$ characterizes the maximum of information for the $\mathrm{N}$ systems. The normalized quantity $\overline{\mathcal{H}}$ is the following $\overline{\mathcal{H}}=\mathcal{H} / \mathcal{H}_{\max }$, then $0 \leq \overline{\mathcal{H}} \leq 1$, where $\mathcal{H}_{\max }=\log \mathrm{N}$.

If the system is out of equilibrium, the entropy $\mathcal{H}$ can be expanded around this maximum $\mathcal{H}_{\max }$ :

$$
\mathcal{H}\left(\mathrm{p}_{1}, \mathrm{p}_{2}, \ldots, \mathrm{p}_{\mathrm{N}}\right)=\log \mathrm{N}-\frac{\mathrm{N}}{2} \sum_{i=1}^{\mathrm{N}}\left(\mathrm{p}_{\mathrm{i}}-\frac{1}{\mathrm{~N}}\right)^{2}+\cdots=\mathcal{H}_{\max }-\frac{\mathrm{N}}{2} \mathrm{D}+\cdots,
$$

where the quantity $D=\sum_{i}\left(p_{i}-1 / N\right)^{2}$ denotes the disequilibrium.

Let us multiply this expansion by $\mathcal{H}$ in the following:

$$
\mathcal{H}^{2}=\mathcal{H} \cdot \mathcal{H}_{\max }-\frac{\mathrm{N}}{2} \mathcal{H} \cdot \mathrm{D}+\mathrm{g}\left(\mathrm{N}, \mathrm{p}_{\mathrm{i}}\right),
$$

where $g\left(N, p_{i}\right)$ contains the entropy multiplied by the rest of Taylor expansion terms, which presents the form $\frac{1}{N} \sum_{i}\left(N p_{i}-1\right)^{m}$ with $m>2$. If we rename $\mathrm{C}=\mathcal{H} \cdot \mathrm{D}:$

$$
\mathrm{C}=\frac{2}{\mathrm{~N}} \cdot \mathcal{H} \cdot\left(\mathcal{H}_{\max }-\mathcal{H}\right)+2 \mathrm{~g} / \mathrm{N} .
$$

This expression shows the connection among the entropy, disequilibrium and complexity.

Let us define the function of disequilibrium $\mathrm{D}$ on the probability distribution $\left\{p_{j}: j=1 \ldots, N\right\}$ in $N$-system.

The Euclidean measure have been used i.e. the quadratic distances from the probability distribution of each symbol sequences $\mathrm{P}\left(\mathrm{O}^{(\mathrm{n})}\right)$ to the equiprobability $\mathrm{P}\left(\mathrm{O}_{e}^{(\mathfrak{n})}\right)$ :

$$
\mathrm{D}=\sum_{i=1}^{\mathrm{N}}\left(\mathrm{p}_{\mathrm{i}}-\mathrm{p}_{e}\right)^{2}, \text { where } \mathrm{p}_{e}=\frac{1}{\mathrm{~N}}
$$

The maximum disequilibrium is reached for dominant symbol series $\mathrm{O}_{\mathrm{c}}^{(\mathfrak{n})}$ with $p_{c} \sim 1$ and $D_{c} \rightarrow 1$ for $N$ is increasing, while the disequilibrium vanishes. i.e. $\mathrm{D} \sim 0$ for $p_{i} \sim 1 / \mathrm{N}$. For any other probability distribution $\mathrm{D}$ will have value between these two extrema. The normalized disequilibrium is $\bar{D}=\mathrm{D} \cdot \mathrm{D}_{e}$, where $\mathrm{D}_{e}$ equals to $\frac{\mathrm{N}}{\mathrm{N}-1}$.

\subsubsection{Measure of statistical complexity}

The family of the complexity measure contains the product of disorder $\mathcal{H}$ and disequilibrium D for different type of the time series. This is interplay 
between the information stored in the system and its disequilibrium. We will define the measure of statistical complexity $\mathrm{C}$ [25] in the following expression in $\mathrm{N}$-system:

$$
\mathrm{C}=\mathcal{H} \cdot \mathrm{D}=-\left(\sum_{i=1}^{\mathrm{N}} \mathrm{p}_{i} \log \mathrm{p}_{i}\right)\left(\sum_{i=1}^{\mathrm{N}}\left(\mathrm{p}_{i}-\frac{1}{\mathrm{~N}}\right)^{2}\right) .
$$

This quantity is larger or equals to zero, i.e. $\mathrm{C} \geq 0$. The normalized value of $\mathrm{C}$ is $\overline{\mathrm{C}}=\overline{\mathcal{H}} \cdot \overline{\mathrm{D}}=(\mathcal{H} / \log \mathrm{N})(\mathrm{D} \cdot(\mathrm{N} /(\mathrm{N}-1)))$.

The definition of statistical complexity measure can be divided into three categories: (i) it is growing with increasing entropy, (ii) it is a convex function and equals to minimum at the $\mathcal{H}=0$ total order and $\mathcal{H}=1$ total disperse state and a maximum at transition level, where the probability distribution is $p_{e}$, (iii) it is decreasing with increasing entropy [29]. We will study the second case in this article.

Because the statistical complexity was defined in a finite system, therefore it depends on the scale. At each scale of observation a new set of accessible symbol sequence $\mathrm{O}^{(\mathfrak{n})}$ appears with its corresponding probability distribution $\mathrm{P}\left(\mathrm{O}^{(\mathrm{n})}\right)$ therefore the complexity changes.

The complexity $\mathrm{C}$ is finite and limiting but it is not necessary a unique function of $\mathcal{H}$, there exists a range of value between a minimal value $C_{\text {min }}$ and a maximal value $C_{\text {max }}$. Thus, evaluating the complexity provides more important additional information regarding the peculiarities of a probability distribution.

Two basic incidents are distinguished in the relationship between the entropy $\mathcal{H}$ and complexity $\mathrm{C}$. On the one hand the time sequence can be found in any of its accessible symbol sequence $\mathrm{O}^{(\mathfrak{n})}$ with the same probability. All of them contribute in equal measure to the information stored. On the other hand, minimal information is enough to describe the system considering some symmetries properties and distance.

It should be noticed that different measures for complexity employed for different probability distribution. Tsallis suggested a generalisation of the Shannon-Boltzmann-Gibbs entropic measure [34] and A. Rényi introduced a definition of entropy for discrete probability distribution in 1950s [31].

The disequilibrium can be extended for various probability distribution. Jensen-Kullback divergence was investigated for relative entropies [29] and Wootters statistical distance was applied for two probability distributions [37], which can be used in the quantum mechanic. 
In Section 5 we study the statistical complexity on the system out of equilibrium by numerical approximation.

\section{Chaotic motion}

In this section we introduce the Lyapunov exponent [8], which characterises the chaotic behaviour of dynamical systems. This quantity is expressed by probability density along the ergodic trajectory [6].

Let us introduce a map $f: \mathbb{R} \rightarrow \mathbb{R}$, where $x_{t+1}=f\left(x_{t}\right)$, which $(t=$ $0,1,2,3 \ldots)$ corresponds to the trajectory $x_{0}, x_{1}, x_{2} \ldots$ at the time series $t=$ $0,1,2 \ldots$ This map contains stable or unstable fixpoints $x^{*}$ and it is differentiable near to the fixpoints $x^{*}$. We can expanded the map $f$ around the fixpoint $x^{*}$ upto linear expression:

$$
\frac{\left|x_{\mathrm{t}+1}-x^{*}\right|}{\left|x_{\mathrm{t}}-x^{*}\right|} \approx\left|\frac{\partial f(x)}{\partial x}\right|_{x^{*}} .
$$

The solution of this equation is written by the next form $\left.\left|x_{\mathrm{t}}-x\right| \approx\left|\frac{\partial \mathrm{f}(\mathrm{x})}{\partial x}\right|_{\chi^{*}}\right|^{\mathrm{t}}=$ $c e^{\lambda t}$, where $c \in \mathbb{R}$ is a constant value. If $\lambda<0$, then the fixpoint $x^{*}$ becomes stable. If $\lambda>0$, then the fixpoint $x^{*}$ turns into unstable, and in the case of $\lambda=0$ the fixpoint $x^{*}$ is marginal stable.

The $f(x)$ map was extended upto first order, therefore we can not determinate the whole trapping region of the fixpoint $x^{*}$.

As a consequence we defined the Lyapunov exponent:

$$
\bar{\lambda}=\lim _{n \rightarrow \infty} \frac{1}{n} \sum_{t=0}^{n} \ln \left|\frac{\partial f(x)}{\partial x}\right|_{x_{t}},
$$

along the trajectories, where these orbits converge to the fixpoint $\chi_{*}$.

The Lyapunov exponent $\bar{\lambda}$ depends on the initial condition of the trajectories, therefore we denote $x_{n}\left(x_{0}\right) \equiv f^{n}\left(x_{0}\right)$ and it can be used as $\bar{\lambda}=$ $\lim _{n \rightarrow \infty} \frac{1}{n} \ln \left|\frac{\partial f^{n}\left(x_{0}\right)}{\partial x_{0}}\right|$.

If $\bar{\lambda}>0$, then the motion is chaotic. The Lyapunov exponent is sensitive to the initial condition. The $\mathrm{d}$ distance of points of the trajectories increases exponentially, where the $x_{0}$ and $x_{0}+\varepsilon$ points $(\varepsilon>0, \varepsilon \in \mathbb{R})$ were near to each other around the unstable fixpoint $x^{*}$ at the first time step $(t=0)$.

Then the form of Lyapunov exponent is the following:

$$
\bar{\lambda} \approx \lim _{n \rightarrow \infty} \frac{1}{n} \ln \left|\frac{f^{n}\left(x_{0}+\varepsilon\right)-f^{n}\left(x_{0}\right)}{\varepsilon}\right|,
$$


where $\varepsilon \rightarrow 0$. This expression can be written $\left|f^{n}\left(x_{0}+\varepsilon\right)-f^{n}\left(x_{0}\right)\right| \approx|\varepsilon| e^{n} \bar{\lambda}$.

The ergodicity plays important role in the chaotic motion.

We consider the ergodic paths, where $h\left(x_{t}\right)$ means an absolute continuous integrable function $(h: \mathbb{R} \rightarrow \mathbb{R}$ ) along the trajectory. At almost all initial conditions $x_{0}$ the average of the function $h\left(x_{t}\right)$ is introduced as following:

$$
\lim _{n \rightarrow \infty} \frac{1}{n} \sum_{t=0}^{n} h\left(x_{t}\right)=\int_{0}^{1} h(x) P(x) d x \equiv \int_{0}^{1} h(x) d \mu(x)
$$

where $\mu(x)$ means some invariant measure, $\frac{\mu(x)}{d x}=P(x)$ is a probability density and the exact form is given by the map $f(x)$. This expression is measure invariant therefore:

$$
\int_{0}^{1} h(x) d \mu(x)=\lim _{n \rightarrow \infty} \frac{1}{n} \sum_{t=0}^{n} h\left(x_{t}\right)=\lim _{n \rightarrow \infty} \frac{1}{n} \sum_{t=0}^{n} h\left(f\left(x_{t}\right)\right)=\int_{0}^{1} h(f(x)) d \mu(x) .
$$

The Lyapunov exponent is defined by the $\mathrm{P}(\mathrm{x})$ probability density along the ergodic orbit:

$$
\bar{\lambda}=\int_{0}^{1} P(x) \ln \left|f^{\prime}(x)\right| d x
$$

In the chaotic motion the strange attractor $S$ plays similar role in the case of aperiodic motion as the attractor in the periodic motion. Let us choose that orbits, which are characterized by probability distribution $P(x)$. The set $F$ contains the initial points of these trajectories and the set $\mathrm{L}$ is defined following $L=\{x \mid P(x)>0\}$. Then we take the union of the set $L$ with its closure. If the Lyapunov exponent is larger then zero on this set, than we gain the strange attractor $S$ and the trapping region corresponds to the closure of the set $F$. The motion comes on this set $S$ after finite iteration and the points of trajectory follow one to the other randomly.

We study the time series of the measurement of length $n \underline{x}^{(n)}$ and the error of initial point $x_{0}^{(\mathfrak{n})}$ is $\varepsilon>0$. Let us suppose $\bar{\lambda}>0$, then the $\varepsilon^{\prime} \approx|\varepsilon| e^{k \bar{\lambda}}$ at the kth element of sequence $x_{k}^{(n)}$ i.e. the error of time series is increasing as $k$ becomes larger, we can not predict the value $x_{k+1}^{(n)}$ more exactly than $\left|\varepsilon^{\prime}\right| e^{\bar{\lambda}}$. Then the value of the ergodic time series becomes unpredictable. Therefore we apply the statistical complexity to determine the location of the strange attractor in the parameter space.

On a computer the study of the dynamical system appears in a finite $\mathrm{m}$ dimensional space, then we have an $m$ dimensional signal $z(t)$. In a physical 
experiment a single scalar variable $\mathfrak{u}(\mathrm{t})$ is monitored for a system, which has an infinite dimensional phase space $\mathcal{M}^{\prime \prime}$.

In this case we restrict our attention to the dynamics on a finite dimension attractor $A$ in the space $\mathcal{M}^{\prime \prime}$. Otherwise we generate several different scalar values $z_{i}(t) i=1 \ldots, \mathcal{N}$ from the original $u(t)$.

The only way to obtain several measurements from a single one is to use time delays. We choose different delays $\mathcal{T}_{1}=0, \mathcal{T}_{2}, \ldots, \mathcal{T}_{\mathcal{N}}$ and it can be written $z_{k}(t)=u\left(t+\mathcal{T}_{k}\right)$. We can generate an $\mathcal{N}$ dimensional signal in this manner.

The successive time derivative of the signal is formed: $z_{k+1}(t)=d^{k} z_{1}(t) / d t^{k}$, but the numerical differentiations produce high level of error. As usually we should measure several experiment signal produce more exactly values.

The reconstruction of the dynamical process provides an $\mathcal{N}$ dimensional image $\pi A$ of an attractor $A$, which has finite Haussdorff dimension and it is embedded in an infinite dimensional space $\mathcal{M}^{\prime \prime}$. The projection will look different according to the choice of variables. Taken proved that theorem (1981): If we use enough variables, typically about twice the Haussdorff dimension, we shall generally get a good projection [8]. This method produces the attractor, but the realisation is difficult.

In the next section we introduce the generalized number system and the set $\mathrm{B}_{\gamma}$, which is a fractal structure [11], but there are periodic motions on it, therefore it is not chaotic.

\section{Generalized number system}

I. Kátai investigated the concept of generalized number systems [18] in the 1970s. This idea is developed on different algebraic structures expansively as real quadratic fields [10], imaginary quadratic fields [19].

We introduce the basic definition in this section according to the literature $[20,11]$.

Let $\mathbb{Z}_{k}$ be a ring of integer vectorial in $\mathbb{R}_{k}(k \geq 1)$. A $k \times k$ type matrix with integer elements is noted by $M$, where $\mathcal{L}=M \mathbb{Z}_{k}$. Then $\mathcal{L}$ is a subgroup in $\mathbb{Z}_{\mathrm{k}}, \mathrm{O}\left(\mathbb{Z}_{\mathrm{k}} / \mathcal{L}\right)=\mathrm{t}$ the order, where $\mathrm{t}=|\operatorname{det} \mathrm{M}|$. We introduce the digit set $\mathcal{A}$ in the following. Let $\mathcal{A}=\left\{\mathrm{a}_{0}=0, \mathrm{a}_{1}, \ldots, \mathrm{a}_{\mathrm{t}-1}\right\}$ mean a complete set of the representation of the residue classes $\bmod M$ for $(t \geq 2)$. We define the number system $(\mathcal{A}, M)$, if each $n \in \mathbb{Z}_{\mathrm{k}}$ can be written by uniqueness expansion form:

$$
\mathrm{n}=\mathrm{a}_{0}+M \mathrm{a}_{1}+\cdots+\mathrm{M}^{\mathrm{h}-1} \mathrm{a}_{\mathrm{h}-1}, \quad \mathrm{a}_{\mathrm{j}} \in \mathcal{A} \text { for } \mathrm{h}>0 .
$$

We define the function $J: \mathbb{Z}_{k} \rightarrow \mathbb{Z}_{k}$, where the ring of integer vectorial is 
mapped to onto itself. There exists a unique $a_{0} \in \mathcal{A}$ and $n_{1} \in \mathbb{Z}_{\mathrm{k}}$ such that $n=a_{0}+M n_{1}$ for every $n \in \mathbb{Z}_{k}$, i.e. let $J(n)=n_{1}$ be.

The set $\mathrm{H}$ plays fundamental role in the number system $(\mathcal{A}, M)$. Let us define $\mathrm{H}$ in the following:

$$
\mathrm{H}=\left\{z \mid z=\sum_{i=1}^{\infty} M^{-i} a_{i}, \quad a_{i} \in \mathcal{A}\right\} .
$$

The set $\mathrm{H}$ is compact. If $(\mathcal{A}, \mathrm{M})$ is a number system, then

$$
\cup_{n \in \mathbb{Z}_{k}}(H+n)=\mathbb{R} .
$$

We say that $(\mathcal{A}, M)$ is just touching covering system (JTCS), if every $n_{1}, n_{2} \in$ $\mathbb{Z}_{\mathrm{k}}, \mathrm{n}_{1} \neq \mathrm{n}_{2}$ :

$$
\lambda\left(\mathrm{H}+\mathrm{n}_{1} \cap \mathrm{H}+\mathrm{n}_{2}\right)=0,
$$

where $\lambda$ is the Lebesques measure. Let $\mathcal{B}=\mathcal{A}-\mathcal{A}=\left\{\mathrm{a}_{\mathfrak{u}}-\mathrm{a}_{v} \mid \mathrm{a}_{\mathfrak{u}}, \mathrm{a}_{v} \in \mathcal{A}\right\}$ hold. We introduce a set $S$ following. That element $\gamma \in \mathbb{Z}_{k}$ is contained in the set $S$, which fulfils $\gamma \neq 0$ and satisfies the following expression:

$$
\mathrm{H} \cap \mathrm{H}+\gamma \neq \emptyset \text {. }
$$

This set is assigned by $B_{\gamma}$ and

$$
\mathrm{B}=\cup_{\gamma} \mathrm{B}_{\gamma} .
$$

More detailed, if $z \in B_{\gamma}$, then $z$ can be extended by this form $z=\sum_{i=1}^{\infty} M^{-i} a_{i}=$ $\gamma+\sum_{i=1}^{\infty} M^{-i} a_{i}^{\prime}$, where $a_{i}, a_{i}^{\prime} \in \mathcal{A}$ and $\gamma=\sum_{i=1}^{\infty} M^{-i} e_{i}$, where $e_{i}=a_{i}-a_{i}^{\prime} \in \mathcal{B}$.

We will determinate the statistical complexity of the finite approximation set $\mathrm{B}_{\gamma}$ on the ring of quadratic integers at a given algebraic number fields in Section 4.

In the next section we investigate a walk along the finite transition graph, which is analogous to dynamical system.

\subsection{Transition graph of number system}

Let us produce a finite directed labeled graph $\mathrm{G}(\mathrm{S})$ according to the article [35], where the function $\mathrm{Q}: S \rightarrow S\left(S \subseteq \mathbb{Z}_{\mathrm{k}} \backslash\{0\}\right)$ means a walk $\mathrm{P}$ along the transition graph.

The elements of the set $S$ correspond to vertices of the graph $G(S)$ and the edges can be defined as follows: 
There exists a directed edge from $\gamma_{k}$ to $\gamma_{k+1}$ and it is labeled by $\delta \in \mathcal{B}$, if $\mathrm{Q}\left(\gamma_{\mathrm{k}}\right)=\gamma_{\mathrm{k}+1}$, i.e. $\gamma_{\mathrm{k}+1}=\gamma_{\mathrm{k}} \mathrm{M}-\delta, \mathrm{k} \in \mathbb{N}$.

Let us construct the graph $\mathrm{G}(\mathrm{S})$ :

The elements $\gamma_{i}$ of the set $S$ can be computed by the following way:

- Initial condition: If the first element $\gamma_{1}$ satisfies $\mathrm{Q}\left(\gamma_{1}\right)=0$, then this element $\gamma_{1}=0$.

- If $\gamma_{2} \in S$ and there exists en edge, which goes from $\gamma_{1}$ to $\gamma_{2}$, than $\gamma_{1} \in S$.

- Each element of the set $S$ fulfils the next condition. That values of outgoing and ingoing degree of the vertices $\gamma_{k}$ are larger than zero i.e. $\operatorname{deg}^{+}\left(\gamma_{k}\right)>$ $0, \operatorname{deg}^{-}\left(\gamma_{k}\right)>0$.

○ The set $S$ contains periodic points: $Q^{r}\left(\gamma_{1}\right)=\gamma_{1}$.

The loop of the algorithm:

- From every $\gamma_{1} \in S$ an edge fits to $\gamma_{2}$, if $\gamma_{2}=\gamma_{1} M-\delta$ for $\delta \in \mathcal{B}$.

$\circ$ If $\operatorname{deg}^{+}\left(\gamma_{1}\right)=0$, than erase the vertices $\gamma_{1}$ and all edges, which directed to $\gamma_{1}$.

Repeat these finite steps, until we delete all those nodes from which no edge goes out i.e. $\operatorname{deg}^{-}\left(\gamma_{k}\right)=0$ or ends, remove all coinciding edges as well.

We obtain the directed transition graph $\mathrm{G}(\mathrm{S})$.

Let $P:=\gamma_{1} \stackrel{\delta_{1}}{\longrightarrow} \gamma_{2} \stackrel{\delta_{2}}{\longrightarrow} \gamma_{3} \ldots, \gamma_{r-1} \stackrel{\delta_{r-1}}{\longrightarrow} \gamma_{r}$ be a walk of length $r$ on the graph $\mathrm{G}(\mathrm{S})$, it is labeled by $\left(\delta_{1}, \delta_{2}, \ldots, \delta_{\mathrm{r}-1}\right)$, i.e. for finite orbit of length $\mathrm{r}$ $\mathrm{Q}^{(\mathrm{r})}\left(\gamma_{1}\right)=\gamma_{\mathrm{r}}$.

Because $z \in \mathrm{B}_{\gamma}$ was defined by expression (8):

$$
z=\sum_{i=1}^{\infty} M^{-i} f_{i}, \quad f_{i} \in \mathcal{A} .
$$

Every infinitely long walk $P$ is assigned by the series of labels: $\delta_{1}, \delta_{2}, \ldots, \delta_{r-1}, \ldots$, where $\delta_{i}=f_{i}-f_{i}^{\prime}$ with appropriate $f_{i}, f_{i}^{\prime} \in \mathcal{A}$. Therefore $z \in B_{\gamma}$ can be labeled by the sequence $f_{1}, f_{2} \ldots$

\section{$5 \quad$ Numerical results}

We introduce an appropriate measure of the time series and the statistical complexity on lattice. 


\subsection{Probability measure and euclidean distance on lattice}

Let us consider a lattice $C^{\prime}$ in the $\mathbb{R}^{\mathrm{d}}$ with linear size $\varepsilon(\varepsilon>0, \varepsilon \in \mathbb{R})$, where $\mathrm{C}_{j}^{\prime}$ assigns the elementary box of lattice $C^{\prime}$ in the following way:

$$
C^{\prime}=\cup_{j} C_{j}^{\prime} \text {, and } C_{j}^{\prime} \cap C_{i}^{\prime}=\emptyset \text {, where } j, i \in\left\{0, \ldots, N^{\prime \prime d}-1\right\} \text {, }
$$

where the set of $C_{j}^{\prime}$ is a partition of $\left[0, N^{\prime \prime} \varepsilon^{[d} \subset \mathbb{R}^{d}\right.$. Let $K$ be a compact set, which contains the measured value $y$ :

$$
\begin{gathered}
\mathrm{T}_{j}=\mathrm{K} \cap \mathrm{C}_{j}^{\prime} \neq \emptyset, \text { where } j=1, \ldots, \mathrm{M}^{\prime} \text { and } \mathrm{K} \cap \mathrm{C}_{j}^{\prime}=\emptyset \text { for any other } \mathrm{C}_{j}^{\prime} . \\
\mathrm{T}=\cup_{j=1}^{\mathrm{M}^{\prime}} \mathrm{T}_{j}, \quad \text { where } \mathrm{T}_{j} \cap \mathrm{T}_{i}=\emptyset, \quad i \neq j .
\end{gathered}
$$

The compact set $K \subset \mathbb{R}^{d}$ consists of every points $x_{k}^{(n)}(k=1, \ldots, n)$ of some orbit of length $n$. Each path corresponds to the series of the indices $j$ for which $x_{k}^{(n)} \in T_{j}$ and $j \in\left\{1, \ldots, M^{\prime}\right\}$.

The lattice size $\varepsilon$ and the unit of the time interval $\Delta t$ equal to a constant.

We define the distance on this lattice, where a box of the linear size $\varepsilon$ is taken as the unit length. We introduce constant values, that is $\mathbf{a}$, which means the minimal distance between two points $a=\min \{|x-y|: x \neq y, x, y \in K\}$ and $\mathrm{L}$, which is the diameter of the set $\mathrm{K}$ i.e. $\mathrm{L}=\max \{|x-y|: x, y \in \mathrm{K}\}$.

Let us introduce $I^{\prime}$, which contains all series of the indices $\underline{\mathfrak{n}}^{\prime}=\left(n_{1}^{\prime}, n_{2}^{\prime} \ldots, n_{n}^{\prime}\right)$, where $n_{1}^{\prime}, n_{2}^{\prime}, \ldots, n_{n}^{\prime} \in\left\{1, \ldots, M^{\prime}\right\}$, and $M^{\prime} \in \mathbb{N}$. The elements of the set $T_{n^{\prime}}^{(n)}$ correspond to the path of length $n$. The set $T_{\underline{n}^{\prime}}^{(\mathfrak{n})}$ is the following:

$$
\mathrm{T}_{\underline{n}^{\prime}}^{(\mathrm{n})}=\left\{\left(x_{1}^{(\mathrm{n})}, x_{2}^{(\mathrm{n})} \ldots, x_{\mathrm{n}}^{(\mathrm{n})}\right) \mid x_{1}^{(\mathrm{n})} \in \mathrm{T}_{\mathrm{n}_{1}^{\prime}}, x_{2}^{(\mathrm{n})} \in \mathrm{T}_{\mathrm{n}_{2}^{\prime}}, \ldots, x_{\mathrm{n}}^{(\mathrm{n})} \in \mathrm{T}_{\mathrm{n}_{\mathfrak{n}}^{\prime}}\right\} .
$$

Analogously to the article [11] we define a measure by the map $\mu\left(T_{\underline{n}^{\prime}}^{(\mathfrak{n})}\right)$ on the lattice:

$$
\mu\left(T_{\underline{n}^{\prime}}^{(\mathfrak{n})}\right)=\frac{\left|T_{\underline{n}^{\prime}}^{(\mathfrak{n})}\right|}{\left|T^{(\mathfrak{n})}\right|}, \quad \text { where } \quad T^{(\mathfrak{n})}=\cup_{\underline{\mathfrak{n}}^{\prime} \in \mathrm{I}^{\prime}}{\underline{\underline{n}^{\prime}}}^{(\mathfrak{n})} .
$$

We note $T_{\underline{m}^{\prime}}^{(n)} \cap T_{\underline{n}^{\prime}}^{(\mathfrak{n})}=\emptyset$, if $\underline{n}^{\prime} \neq \underline{m}^{\prime}, \underline{n}^{\prime}, \underline{m}^{\prime} \in I^{\prime}$ and $1<\left|I^{\prime}\right| \leq M^{n}$ and

$$
\sum_{\underline{n}^{\prime} \in I^{\prime}} \frac{\left|T_{\underline{n}^{\prime}}^{(n)}\right|}{\left|T^{(n)}\right|}=1
$$


The measure of Shannon entropy is defined for finite time series on lattice:

$$
\tilde{\mathcal{H}}=-\sum_{\underline{n}^{\prime} \in I^{\prime}} \mu\left(T_{\underline{n}^{\prime}}^{(\mathfrak{n})}\right) \ln \mu\left(T_{\underline{n}^{\prime}}^{(\mathfrak{n})}\right) .
$$

We investigate the measure of disequilibrium on grid:

$$
\tilde{\mathrm{D}}=\sum_{\underline{\mathrm{n}}^{\prime} \in \mathrm{I}^{\prime}}\left(\mu\left(\mathrm{T}_{\underline{\underline{n}}^{\prime}}^{(\mathrm{n})}\right)-\frac{1}{\mathrm{~N}}\right)^{2} .
$$

Let us introduce the measure of complexity is the following on the lattice:

$$
\tilde{\mathrm{C}}=\tilde{\mathcal{H}} \tilde{\mathrm{D}} .
$$

In the next section we determine the statistical complexity $\tilde{C}$ for the Lozi map on some range of parameter $a, b$ and for the finite approximation of the set $\mathrm{B}_{\gamma}$.

\subsection{Approximation of the statistical complexity}

In this section we consider the indicator role of the statistical complexity i.e. it enables to point out the nonlinearity on the time series $x_{1}^{(\mathrm{n})}, \ldots, x_{n}^{(\mathrm{n})}$. The Lozi map possesses chaotic behaviour in some ranges of parameter and initial condition, but it is not everywhere differentiable, therefore we perform numerical approximation. These properties were discussed by bifurcations [3] and the Lypunov exponents [9], but the statistical complexity can be determined more easier than the other quantities.

We present the statistical complexity on the finite approximation of the set $\mathrm{B}_{\gamma}$, which is obtained for a generalized number system in quadratic integer.

\subsubsection{Lozi map}

R. Lozi introduced a two dimensional a piecewise linear map [27], which assigns the plane into itself $f:(\mathbb{R} \times \mathbb{R}) \rightarrow(\mathbb{R} \times \mathbb{R})$, it is a homomorphism on a metric space:

$$
f(x, y)=(1+y-a|x|, b x) .
$$

A numerical simulation is plotted on the Figure 1.

M. Misiurewicz [30] proved that Lozi map has a strange attractor on some set of values $a, b$, which arises from the intersection of the images of the trapping region. He supposed six conditions: 


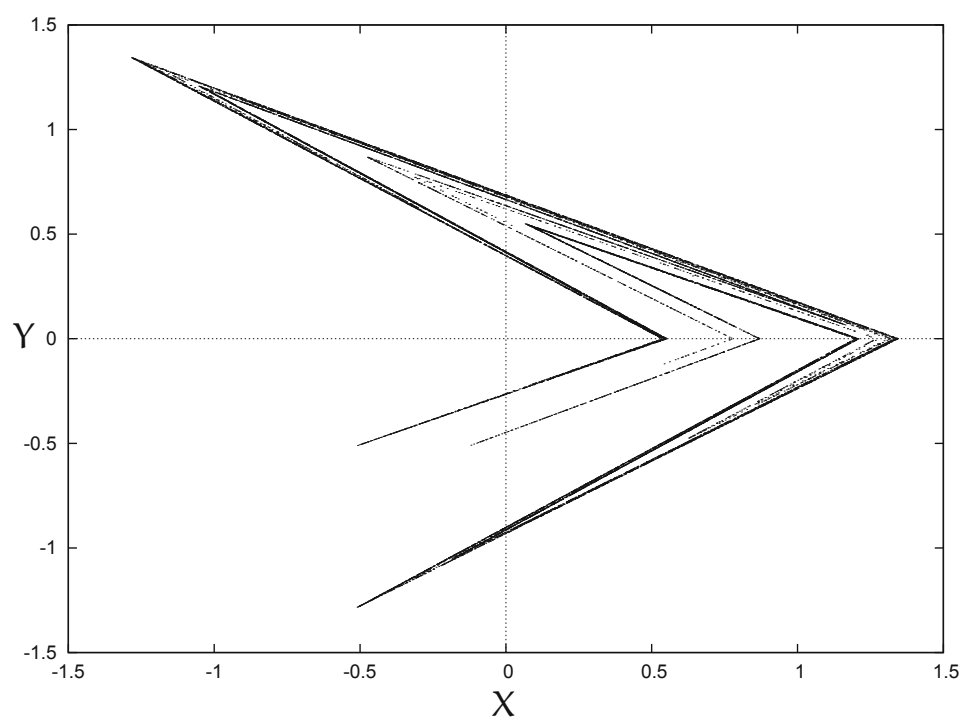

Figure 1: Lozi map $\left(a=1.7, b=0.5, N^{\prime}=10^{4}\right.$ iteration $)$
1. $0<\mathrm{b}<1, \mathrm{a}>0$
2. $a>b+1$
3. $2 a+b<4$
4. $a>\frac{1}{2} \sqrt{3 b^{2}+4+\sqrt{\left(3 b^{2}+4\right)^{2}-32 b}}$
5. $\mathrm{b}<\frac{\mathrm{a}^{2}-1}{2 \mathrm{a}-1}$
6. $a \sqrt{2}>b+2$.

His theorem was proven by the geometrical verification:

That set, which satisfies these assumptions (1-6) is open and non-empty.

If the parameters fulfil the first and $a+b>1$ conditions, the map $f$ has two hyperbolic fixpoints: $F 1=(1 /(1+a-b), b /(1+a-b))$ and $F 2=(1 /(1-$ $a-b), b /(1-a-b))$,

The stable and unstable manifold of these points $W_{\mathrm{F} 1}^{\mathrm{u}}, \mathrm{W}_{\mathrm{F} 1}^{\mathrm{s}}, \mathrm{W}_{\mathrm{F} 2}^{\mathrm{u}}$ and $W_{\mathrm{F} 2}^{\mathrm{s}}$ located on the plane $(X, Y)$ according to the eigenvectors of the map $f$. The nonempty set, which satisfies the conditions 1-3 corresponds to the trapping region. Because the strange attractor equivalents to closure of unstable manifolds, he justified that subset of trapping regions, which fulfils the first and 3-5 criterion, suits to the strange attractor.

Let us consider the statistical complexity of Lozi map by numerical approximation. Particularly we study the the strange attractor on the plane of the parameter space $(a, b)$, where $a, b \in \mathbb{R}$ and the relationship between $\tilde{\mathcal{H}}$ and $\tilde{\mathrm{C}}$.

We applied the generalized partition of the Lozi map on the two dimensional lattice $(X, Y)$. The kth element $x_{k}^{(n)}$ of time sequence of length $n$ equals to 1 , if $y>0$, otherwise $x_{k}^{(n)}=0$. 


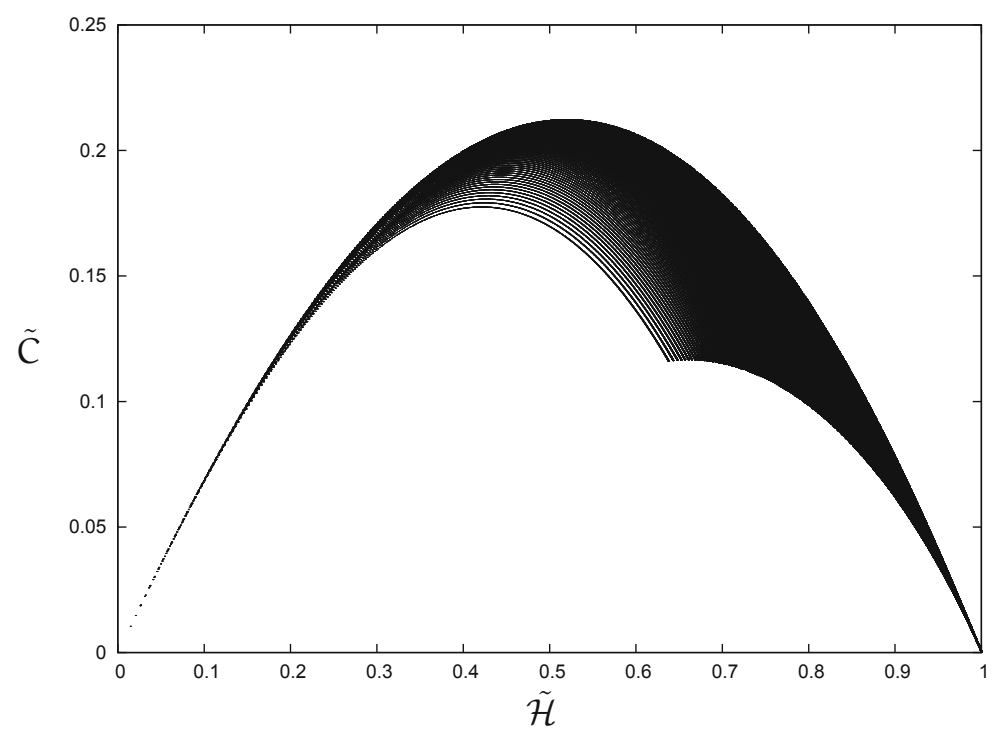

Figure 2: The points of the relationship $\tilde{\mathcal{H}} \times \tilde{C}$ for the ideal gas with uniform probability distribution $(\mathrm{N}=3)$

The time series of length $N^{\prime}\left(N^{\prime}>>n\right)$ is generated by the iteration of the Lozi map and we created an appropriate $\mathrm{N}$-system according to the probability distribution $\left\{p_{1}, \ldots, p_{N}\right\}$ of orbits of length $n \underline{x}^{(n)}$. Then there are $N$ different paths of length $n\left\{\underline{x}_{1}^{(n)}, \underline{x}_{2}^{(n)}, \ldots, \underline{x}_{N}^{(n)}\right\}$, which corresponds to the set of the probability distribution $\left\{p_{1}, \ldots, p_{N}\right\}$, where $p_{l}:=P\left(\underline{x}_{l}^{(n)}\right)(l=1, \ldots, N)$. It is applied to calculate the entropy $\tilde{\mathcal{H}}$, disequilibrium $\tilde{\mathrm{D}}$ and the statistical complexity $\tilde{\mathrm{C}}$ by appropriate measure on lattice.

Before discussing for the statistical complexity of the Lozi map we should mention that system, where the complexity does not have any intricately structure and $\tilde{\mathrm{C}}_{\max } \neq \tilde{\mathrm{C}}_{\min }$, i.e. all possible value of the discrete probability distribution appears, then the points show uniformly dispersion on the plane $\tilde{\mathcal{H}} \times \tilde{C}$ between $\tilde{\mathrm{C}}_{\min }$ and $\tilde{\mathrm{C}}_{\max }$ (Figure 2).

In contract the structure of statistical complexity for the Lozi map is characterized by the intricate dynamics on the plane $\tilde{\mathcal{H}} \times \tilde{C}$ in the range $a \in(0: 2.5)$ and $b \in(0: 1)$ (Figure 3).

It suggests that the structure of relationship between the entropy $\tilde{\mathcal{H}}$ and complexity $\tilde{C}$ becomes more entanglement for chaotic dynamics.

We distinguish 3 different regions of the spectrum following: 


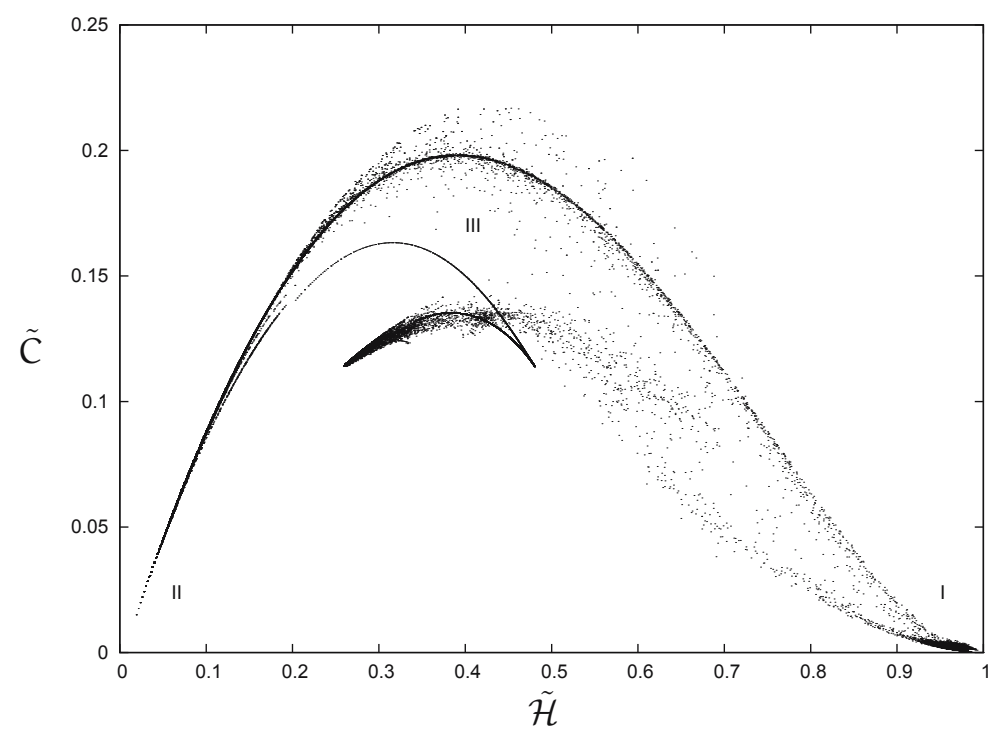

Figure 3: The $\tilde{C}(\tilde{\mathcal{H}})$ spectrum of the Lozi map $\left(n=10, N^{\prime}=1024\right)$

I. The complexity $\tilde{\mathrm{C}} \sim 0$, the entropy $\tilde{\mathcal{H}} \sim 1$ : This subset corresponds to strange attractor. It is characterized by maximal entropy storage near to the equiprobable distribution $\tilde{\mathrm{D}} \sim 0$.

II. We distinguish that region, where the $\tilde{C} \sim 0, \tilde{\mathcal{H}} \sim 0, \mathrm{D} \sim 1$, in this case the value of entropy decreases to zero for the large amount of order.

III. Between the two extreme states (I), (II) the complexity satisfies the maximal value, which corresponds to the transition states.

The statistical complexity $\tilde{C}$ is plotted on the plane of parameter space $(a, b)$ (Figures 4$)$. Suitably for the case I. that region, where the value of the complexity steeply decreases to $\tilde{C} \sim 0$, at the same time the entropy increases to $\tilde{\mathcal{H}} \sim 1$ and the disequilibrium becomes to $\tilde{D} \sim 0$, corresponds to strange attractor i.e. this set is characterized by maximal entropy storage near to the equiprobable distribution.

The result of M. Misiurewitz can be compare with numerical simulation of Lozi map. These are equivalent to each other inside error.

Let us choose $\Delta \tilde{\mathrm{C}} \sim \pm 0.01$ then $\Delta \mathrm{a}, \Delta \mathrm{b} \sim 0.03$ holds i.e. the triangle, which is bounded by the lines to suit the conditions $1,3,6$. Then the measured value $\tilde{\mathrm{C}} \sim 0, \tilde{\mathcal{H}} \sim 1$ corresponds to the theoretical consideration within a given accuracy. This region equivalents to strange attractor (Figure 4.).

We study the finite $\mathrm{N}$-system on lattice, therefore we need to discuss the effect of the scaling properties. If we increase the value of $\mathrm{N}$, then the complexity- 


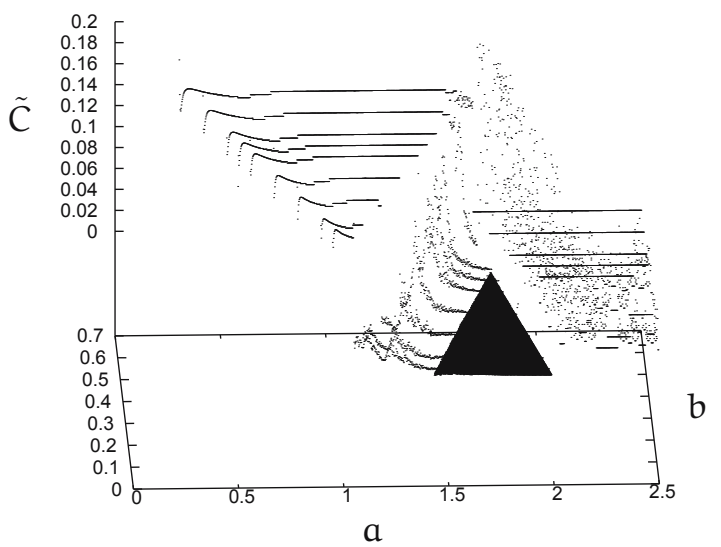

Figure 4: The statistical complexity $\tilde{C}$ of the Lozi map on the parameter plane $(a, b)$

curve peak goes to smaller entropy values in the numerical simulations. It means that the biggest complexity can be reached for less entropy with larger discrete probability distribution $\left\{p_{1}, \ldots, p_{N}\right\}$.

\subsubsection{Finite approximation of $\mathrm{B}_{\gamma}$}

We introduced the idea of the generalized number system for $\mathbb{Z}_{k}$ in the Section 4. It can be extended to the ring of the integer $\mathbb{Z}[\theta]$ in $\mathbb{Q}[\theta]$, where $\theta$ is an algebraic integer and the element of the set forms $f(\theta)=v_{0}+v_{1} \theta+\cdots+$ $v_{n-1} \theta^{n-1}, v_{j} \in \mathbb{Z}$. The equivalency between the $\mathbb{Z}_{k}$ and $\mathbb{Z}[\theta]$ was proven [18]. The digit set is denoted by $\mathcal{A}=\left\{\mathrm{a}_{0}, \mathrm{a}_{0}, \ldots, \mathrm{a}_{\mathrm{t}-1}\right\}(\subset \mathbb{Z}[\theta])$.

The map $\mathrm{J}: \mathbb{Z}[\theta] \rightarrow \mathbb{Z}[\theta]$ is introduced by $\mathrm{J}(\alpha)=\alpha_{1}$, where there exists a unique $\mathrm{b} \in \mathcal{A}$ in $(\mathcal{A}, \theta)$ and a unique $\alpha_{1} \in \mathbb{Z}[\theta]$, where $\alpha=\mathrm{b}+\theta \alpha_{1}$. It can be extended for $\alpha$ by this expression $\alpha_{l}=J^{(l)}(\alpha)$.

Kátai I. and Szabó J proved that $(\theta, \mathcal{A})$ is a canonical number system if and only if $\mathfrak{R} \theta<0$ and $\mathfrak{I} \theta= \pm 1$, where $\theta$ is a Gaussian complex integer and $\mathcal{A}=\{0,1, \ldots, \mathrm{N}(\theta)-1\}(\mathrm{N}(\theta)=\theta \bar{\theta})[21]$.

According to the expression of the fundamental set $\mathrm{H}$ (5), it holds in this extension field $\mathbb{Z}[\theta]$. Let $\rho=1 / \theta$, where $\rho \in \mathbb{C}, 0<|\rho|<1$ and $\mathcal{A}=\{0,1\}$. 
Then the analogue set $\mathrm{H}$ :

$$
\mathrm{H}=\left\{z \mid z=\sum_{i=1}^{\infty} \rho^{i} \mathrm{f}_{i}, \quad \mathrm{f}_{\mathrm{i}} \in \mathcal{A}\right\} .
$$

Because $\mathrm{B}_{\gamma}=\mathrm{H} \cap \mathrm{H}+\gamma$

$$
\mathrm{B}_{\gamma}=\{z \mid z \in \mathrm{H}, z-\gamma \in \mathrm{H}\} .
$$

Therefore all expansions of $\gamma$ appear as

$$
\gamma=\rho^{1} e_{1}+\rho^{2} e_{2} \ldots
$$

where $e_{1}, e_{2} \cdots \in \mathcal{B}=\mathcal{A}-\mathcal{A}$. Because $e_{i}=f_{i}-f^{\prime}{ }_{i}$ holds, where $f_{i}, f^{\prime}{ }_{i} \in \mathcal{A}$, $(i=1 \ldots)$, we can determine all of possible values of the digit $f_{i}$, which follows from expressions (17), (18):

$$
z=\rho^{1} f_{1}+\rho^{2} f_{2} \ldots
$$

The elements of the set $\mathrm{B}_{\gamma}$ contains $z$ over infinite sums, we will approximate them with finite sums. This set is denoted by $\widetilde{B}_{\gamma}$, which contains these elements for some fixed $k$ and $\gamma$, it is written as

$$
x=\sum_{i=1}^{k} \rho^{i} f_{i}, \quad f_{i} \in \mathcal{A} .
$$

The Kolmogorov entropy and the fractal dimension of the finite approximation of the set $\mathrm{B}_{\gamma}$ were published [14] [11].

In the next section we will study the statistical complexity for the set $\widetilde{B}_{\gamma}$, whose every element corresponds to a subset of $\mathrm{B}_{\gamma}$.

\subsubsection{Statistical complexity of the set $\tilde{B}_{\gamma}$}

In this section we present the numerical results, which is obtained for a generalised number system in quadratic integers.

In the article [17] it was proven that $\theta \in \mathbb{C}$ is a root of the polynomial of second-degree $f(x)$, whose coefficients are $a_{2}=1, a_{1}=0, \pm 1, \pm 2, a_{0}=2$. The smallest ring is $\Delta=\{1, \Theta\}$. Then

$$
\cup_{\gamma \in \Delta}(\mathrm{H}+\gamma)=\mathbb{C}
$$




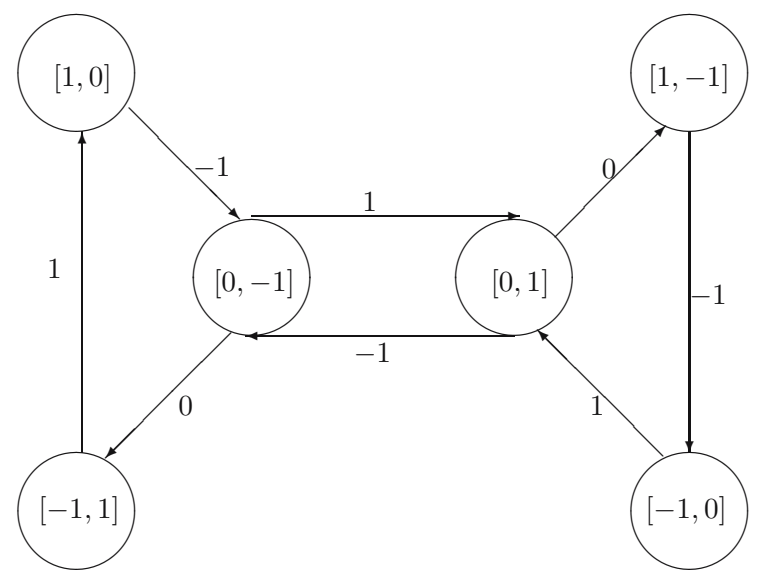

Figure 5: Transition graph for $\theta=-1-i$ and $\mathcal{B}=\{-1,0,1\}$

$$
\lambda\left(\left(H+\gamma_{1}\right) \cap\left(H+\gamma_{2}\right)\right)=0, \quad \gamma_{1} \neq \gamma_{2}, \quad \gamma_{1}, \gamma_{2} \in \Delta
$$

where $\gamma=\sum_{v=0}^{l} a_{v} \Theta^{v}$ and $a_{v} \in \mathcal{A}$. Then $(\Theta, \mathcal{A})$ is a canonical number system in a quadratic field extensions for all these $\Theta$ values.

We construct the transition graph G(S) (Figure 5) according to Section 4.1. The base of the number system is chosen as $\theta=-1-i$ and the digit set $\mathcal{A}=\{0,1\}$. The edge is labeled by an element of the set $\mathcal{B}=\{-1,0,1\}$.

The steps of graph construction are the following:

○ Every $\gamma \in \mathbb{Z}[\Theta]$ which satisfies the condition $|\gamma| \leq \sqrt{2}+1$, we determinate $\eta=\gamma \Theta-\delta$ for $\delta \in \mathcal{B}$. A directed edge fits from $\gamma$ to $\eta$, if $|\eta| \leq \sqrt{2}+1$ holds.

- That $\gamma$ vertices is deleted, which has no edge from $\gamma$ and remove all edges which are directed to $\gamma$.

The process results the graph $\mathrm{G}(\mathrm{S})$.

Let us consider the process of the graph walking $\mathrm{P}$.

- First step in the initial condition we choose one vertex along the graph $\mathrm{G}(\mathrm{S})$ randomly.

$\circ$ The basic concept of the graph walking $P$ is the following. It contains all of possible edges at least ones $\left(\delta_{1}, \delta_{2}, \ldots, \delta_{k}\right)$, i.e. this step produces the minimal length orbit.

- Let us take into consideration, that the outgoing degree of vertices q can be larger than 1 and the graph walking need to contain all edges, therefore the same node can appear more times resulting the perfect series of all directed ones. 


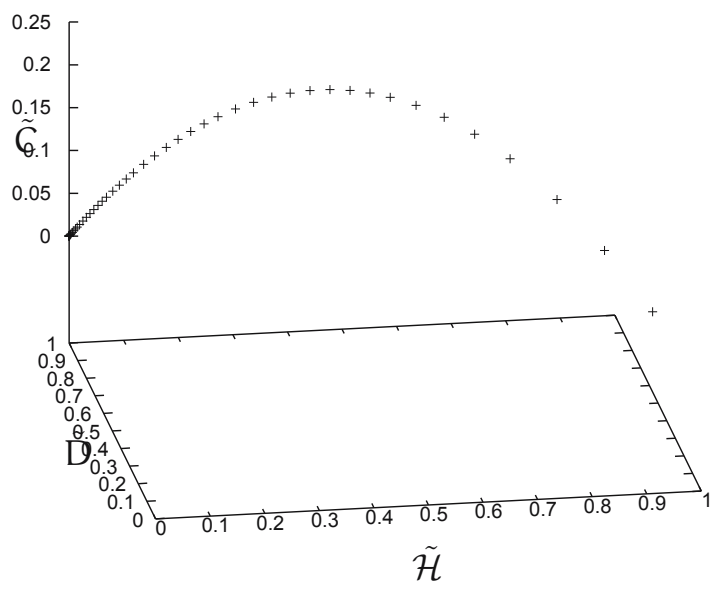

Figure 6: The spectrum $\tilde{\mathrm{C}}(\tilde{\mathcal{H}}, \tilde{\mathrm{D}})$ of the set $\tilde{\mathrm{B}}_{\gamma}\left(\mathrm{N}^{\prime}=8064, \mathrm{n}=50\right)$

○ We need to consider each of sequences $f_{1}, \ldots, f_{r},\left(f_{i} \in \mathcal{A}\right)$ to the series of labels of edges.

The $\mathrm{N}$-system is determined by finite walk on the transition graph $\mathrm{G}(\mathrm{S})$. The probability distribution $\left\{\mathrm{p}_{1}, \mathrm{p}_{2}, \ldots, \mathrm{p}_{\mathrm{N}}\right\}$ is created according to the path of length $n \underline{x}^{(n)}$ along the walk of length $N^{\prime}$.

We determined the Shannon entropy $\tilde{\mathcal{H}}$, the disequilibrium $\tilde{D}$ and the statistical complexity $\tilde{\mathrm{C}}$ on lattice, which is plotted on the Figure 6 .

The points of the curve in three dimensional space $\tilde{\mathcal{H}} \times \tilde{D} \times \tilde{C}$ does not contain any intrinsic structure i.e. any structural complexity and it is a unique convex function $\tilde{C}(\tilde{D})$ respectively $\tilde{C}(\tilde{\mathcal{H}})$. The curve corresponds to the maximal limit of the statistical complexity $\tilde{\mathrm{C}}_{\text {max }}$ of the curve in Figure 3 . on the range $\tilde{\mathcal{H}} \in$ $[0,1]$ and $\tilde{D} \in[0,1]$.

The transition graph, which was introduced analogues to dynamical process on the set $\tilde{\mathrm{B}}_{\gamma}$ points to regular motion.

\section{Summary}

The statistical complexity is an indicator, which can be used to reveal the dynamical behaviour of the finite system. We calculated the complexity $\tilde{C}$ of 
Lozi map on the plane of parameter $(a, b)$, this map has chaotic range, where the strange attractors can be unique specified by complexity $\tilde{C}$, entropy $\tilde{\mathcal{H}}$ and disequilibrium $\tilde{D}$ on lattice. Here the value of entropy becomes $\tilde{H} \sim 1$, the quantity of disequilibrium is $\tilde{\mathrm{D}} \sim 0$, therefore the value of $\tilde{\mathrm{C}}$ changes to 0 sharply on the parameter space. Contrary to the statistical complexity $\tilde{C}$ of the set $\tilde{B}_{\gamma}$ in the generalized number system corresponds to unique function of the entropy $\tilde{\mathcal{H}}$ and disequilibrium $\tilde{D}$ and it fits to the upper limit of the spectrum $\tilde{\mathrm{C}}(\mathcal{H})$ for the Lozi map. Because the chaotic motion on the strange attractor contains aperiodic orbits in contrast to the dynamical behaviour of the set $\mathrm{B}_{\gamma}$ includes periodic paths, the statistical complexity is able to indicate the different properties of time series and it is localised in the finite dimensional space.

\section{References}

[1] C. Adami, N. T. Cerf, Physical complexity of symbolic sequences, Physica D 137, 1-2 (2000) 62-69. $\Rightarrow 230$

[2] C. Anteneodo, A. R. Plastino, Some features of the López-Ruiz-Manchini-Calbet (LMC) statistical measure of complexity, Physics Letters A 223, 5 (1996) 348$354, \Rightarrow 231$

[3] V. Botella-Soler, J. M. Castelo, J. A. Oteo, J. Ros, Bifurcations in the Lozi map, Journ. Phys. A: Math. Theor. 44, 30 (2011) 305101-305115. $\Rightarrow 241$

[4] X. Calbet, R. Lopez-Ruiz, Extremism complexity distribution of a monodimensional ideal gas out of equilibrium, Physica A 382, 2 (2007) 523-530. $\Rightarrow 230$

[5] X. Calbet, R. Lopez-Ruiz, Extremum complexity in the monodimensional ideal gas: The piecewise uniform density distribution approximation, Physica A $\mathbf{3 8 8}$, 20 (2009) 4364-4378. $\Rightarrow 230$

[6] P. Collet, J. P. Eckmann, Iterated maps in the interval as dynamical systems, Birkhäuser, 1980. $\Rightarrow 235$

[7] J. P. Crutchfield, K. Young, Inferring statistical complexity, Phys. Rev. Lett. 63, 2 (1989) 105-108. $\Rightarrow 230$

[8] J. P. Eckmann, D. Ruelle, Ergodic theory of chaos and strange attractors, Rev. of Modern Physics 57, 3 (1985) 617-656. $\Rightarrow 235,237$

[9] Z. Elhadj, J. C. Sprott, A Unified Piecewise Smooth Chaotic Mapping that Contains the Hénon and the Lozi Systems, Annual Review of Chaos Theory, Bifurcations and Dynamical Systems 1 (2012) 50-60. $\Rightarrow 241$

[10] G. Farkas, Number systems in real quadratic fields, Annales Univ. Sci. Rolando Eötvös Budapest. Sect. Comput. 18 (1999) 47-49. $\Rightarrow 237$

[11] G. Farkas, A. Fülöp, The sandbox method in quadratic fields, Annales Univ. Sci. Rolando Eötvös Budapest. Sect. Comput. 28 (2008) 235-248. $\Rightarrow 237,240$, 246 
[12] G. Feng, S. Song, P. Li, A statistical measure of complexity in hydrological systems, Hydr. Eng. Chin. 11 (1998) 14. $\Rightarrow 230$

[13] G. L. Ferri, F. Pennini, A. Plastino, LMC-complexity and various chaotic regime, Physics Letters A 373, 26 (2009) 2210-2214. $\Rightarrow 231$

[14] Á. Fülöp, Estimation of the Kolmogorov entropy in the generalized number system, Annales Univ. Sci. Budapest Sect. Comp. 40 (2013) 245-256. $\Rightarrow 231$, 246

[15] C. M. Gonzalez, H. A Larrondo, O. A. Rosso, Statistical complexity measure of pseudorandom bit generators, Physica A 354 (2005) 281-300. $\Rightarrow 230$

[16] P. Grassberger, Toward a Quantitative Theory of Self-Generated Complexity, Int. Journ. Theor. Phys. 25, 9 (1986) 907-938. $\Rightarrow 231$

[17] K. H. Indlekofer, I. Kátai, P. Racskó, Some remarks in generalized number systems, Acta Sci. Math. 57 (1993) 543-553. $\Rightarrow 246$

[18] I. Kátai, Generalized number systems and fractal geometry, Univ. Janus Pannonius Pécs, 1995. $\Rightarrow 237,245$

[19] I. Kátai, Number systems in imaginary quadratic fields, Annales Univ. Sci. Roland Eötvös Budapest, Sect. Comput. 14 (1994) 91-103. $\Rightarrow 237$

[20] I. Kátai, Generalized number systems in Euclidean spaces, Math. and Computer Modelling 38, 7-9 (2003) 883-892. $\Rightarrow 237$

[21] I. Kátai, J. Szabó, Canonical number systems for complex integers, Acta Sci. Math. 37, 3-4 (1975) 255-260. $\Rightarrow 245$

[22] A. N. Kolmogorov, Entropy per unit time as a metric invariant of automorphism, Doklady of Russian Academy of Sciences, 124 (1959) 754-755. $\Rightarrow 230$

[23] P. T. Landsberg, J. S. Shiner, Disorder and complexity in an ideal nonequilibrium Fermi gas, Phys. Lett. A 245, 3-4 (1998) 228-232. $\Rightarrow 230$

[24] A. Lempel, J. Ziv, On the complexity of finite sequences, IEEE Trans. Inform. Theory 22, 1 (1976) 75-81. $\Rightarrow 230$

[25] R. López-Ruiz, H. L. Mancini, X. Calbet, A statistical measure of complexity, Phys. Letters A 209, 5-6 (1995) 321-326. $\Rightarrow 230,231,234$

[26] M. Lovallo, V. Lapenna, L. Telesca, Transition matrix analysis of earthquake magnitude sequences, Chaos, Soliton and Fractals 24, 1 (2005) 33-43. $\Rightarrow 231$

[27] R. Lozi, Un attracteur étrange du type attracteur de Hénon, Journal de Physique 39 (1978) C5-9. $\Rightarrow 241$

[28] M. T. Martin, A. Plastino, O. A. Rosso, Statistical complexity and disequilibrium, Physics Letters A 311, 2-3 (2003) 126-132. $\Rightarrow 231$ 
[29] M. T. Martin, A. Plastino, O.A. Rosso, Generalized statistical complexity measures: Geometrical and analytical properties, Physica A 369, 2 (2006) 439-462. $\Rightarrow 231,234$

[30] M. Misiurewicz, Strange attractors for the Lozi mappings, Annals of the New York Academy of Sciences 357 (1980) 348-358. $\Rightarrow 241$

[31] A. Rényi, Probability Theory, North-Holland, Amsterdam, 1970. $\Rightarrow 234$

[32] P. T. Saunders, M. W. Ho, On the increase in complexity in Evolution II. The relativity of complexity and the principle of minimum increase, Journ. Theor. Biol. 90, 4 (1981) 515-530. $\Rightarrow 231$

[33] C. E. Shannon, The Mathematical Theory of Communication, Bell System Technical Journal 27 (1948) 379-423, 623-656. $\Rightarrow 232$

[34] C. Tsallis, Possible generalization of Boltzmann-Gibbs statistics, J. Stat. Phys. 52, 1-2 (1988) 479-487. $\Rightarrow 234$

[35] J. M. Thuswaldner, Fractal and number systems in real quadratic number fields, Acta Math. Hungary 90, 3 (2001) 253-269. $\Rightarrow 238$

[36] Z. Yu, G. Chen Rescaled range and transition matrix analysis of DNA sequences, Comm. Theor. Phys. 33, 4 (2000) 673-678. $\Rightarrow 231$

[37] W. K. Wooters, Statistical distance and Hilbert space, Phys. Rev. D 23, 2 (1981) $357-362 . \Rightarrow 234$ 